\title{
Systematic design of high-Q prestressed micro membrane resonators
}

\author{
Gao, Wenjun ; Wang, Fengwen; Sigmund, Ole
}

\section{Published in:}

Computer Methods in Applied Mechanics and Engineering

Link to article, DOI:

10.1016/j.cma.2019.112692

Publication date:

2020

Document Version

Peer reviewed version

Link back to DTU Orbit

Citation (APA):

Gao, W., Wang, F., \& Sigmund, O. (2020). Systematic design of high-Q prestressed micro membrane resonators. Computer Methods in Applied Mechanics and Engineering, 361, [112692].

https://doi.org/10.1016/j.cma.2019.112692

\section{General rights}

Copyright and moral rights for the publications made accessible in the public portal are retained by the authors and/or other copyright owners and it is a condition of accessing publications that users recognise and abide by the legal requirements associated with these rights.

- Users may download and print one copy of any publication from the public portal for the purpose of private study or research.

- You may not further distribute the material or use it for any profit-making activity or commercial gain

- You may freely distribute the URL identifying the publication in the public portal

If you believe that this document breaches copyright please contact us providing details, and we will remove access to the work immediately and investigate your claim. 


\title{
Systematic design of high- $Q$ prestressed micro membrane resonators
}

\author{
Wenjun $\mathrm{Gao}^{1, \mathrm{a},{ }^{*}, \text { Fengwen } \text { Wang }^{2} \text {, Ole Sigmund }}{ }^{2}$ \\ ${ }^{1}$ State Key Laboratory of Disaster Reduction in Civil Engineering, Tongji University, Shanghai 200092, China. \\ ${ }^{a}$ This work was carried out while Wenjun Gao was a visiting student at Department of Mechanical Engineering, Technical University \\ of Denmark. \\ ${ }^{2}$ Department of Mechanical Engineering, Solid Mechanics, Technical University of Denmark, 2800 Kgs. Lyngby, Denmark. \\ *Corresponding author. \\ E-mail address: \\ 2014Joker@tongji.edu.cn (Wenjun Gao); fwan@mek.dtu.dk (Fengwen Wang); sigmund@mek.dtu.dk (Ole Sigmund);
}

\begin{abstract}
Quality factor $(Q)$ and frequency-quality factor $(Q f)$ of prestressed membrane resonators are enhanced by topology optimization. The optimization targets the fundamental mode which is more easily identified in physical experiments compared to high-order modes embedded in possibly dense frequency ranges. Both intrinsic and extrinsic losses are considered in the optimization process. With different combinations of the two damping sources, topology optimization yields distinct designs, which reveals that damping mechanisms significantly affect the optimal geometry of micro resonators. For optimized designs, the $Q f$ in the fundamental mode exceeds the minimum requirement for room-temperature quantum optomechanics $\left(6.2 \times 10^{12} \mathrm{~Hz}\right)$ and reaches $10^{13} \mathrm{~Hz}$ in numerical experiments.
\end{abstract}

Keywords: topology optimization, prestressed membranes, resonators, quality factor, frequency-quality factor

\section{Introduction}

Optomechanical systems are utilized in optomechanical experiments that push several frontiers of contemporary physics, such as quantum-limited accelerometers [1], optomechanics in 3D microwave cavities [2], microwave-to-optical wavelength conversion [3], and quadratic coupling in cavity optomechanics [4]. In these systems, mesoscopic mechanical resonators with ultrahigh quality factors play an important role in coupling light to mechanical motion. However, experiments involving this field have so far been restricted by the need for cryogenic precooling [5,6]. One possible way to overcome cryogenic temperatures and make realistic quantum experiments feasible at room temperature $(298 \mathrm{~K})$ is to enhance the mechanical frequencyquality factor $Q f$ to a regime where $Q f>k_{\mathrm{B}} T_{\text {room }} / h \approx 6.2 \times 10^{12} \mathrm{~Hz}$ ( $h$ is Planck's constant, and $k_{\mathrm{B}}$ is the Boltzmann constant, $T_{\text {room }}$ is the room temperature) [7,8]. This regime will allow for ground state cooling using the radiation pressure force and experiments operating in the quantum domain.

The most promising mechanical resonators for the purposes of quantum optomechanical experiments are devices in the form of high-stress silicon nitride $\left(\mathrm{Si}_{3} \mathrm{~N}_{4}\right)$ strings [9], beams [10] and membranes [4]. Even though it is understood that the prestress dilutes the dissipation intrinsic to the material (or its surfaces) [1113], few resonators under conventional designs can achieve $Q f>6.2 \times 10^{12} \mathrm{~Hz}$ in the fundamental mode at room temperature. If it is very hard to exceed this lower limit for $Q f$ in the fundamental mode, exploring higher $Q f$ in high-order modes could be a straightforward attempt. At least, the frequency can reach a high value. Hitherto, higher $Q f$ has been demonstrated in high-order modes [14,15], but these modes are embedded in a dense frequency range full of neighboring modes that make the dynamic background noisy and intractable. To avoid disturbance from adjacent modes, prestressed trampoline-like resonators focusing on the fundamental mode outperform other conventional designs, reaching a relatively higher $Q>1.0 \times 10^{7} \mathrm{~Hz}$ $[16,17]$. Trampoline-like resonators are featured by a square central pad suspended by four thin highly tensioned tethers, providing a mismatch mechanism to reduce the radiation losses at the clamping points 
supported by a very thick silicon substrate as shown in Fig. 1. The mechanical properties of the resonators are monitored by reflecting a focused laser off from the central pad.

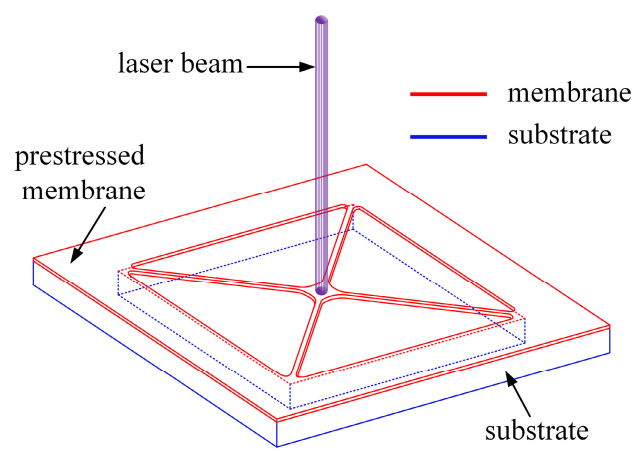

Fig. 1. A schematic of an advanced trampoline-like resonator reported by Norte et al. [17] and Reinhardt et al. [16].

Compared to conventional square membrane resonators, these trampoline-like resonators indicate that structural topology, including various shapes and sizes, directly affects damping distribution and other structural dynamic characteristics, such as frequencies, mass, structural stiffness and especially $Q$ and $Q f$. The impact of geometry on dissipation was also reported by Candler et al. [18]. It is evident that existing designs of resonators can be improved through topology optimization, which is a computational methodology for optimizing the distribution of material in a given design domain. Various numerical formulations and approaches have been proposed throughout the last three decades since the method was conceived by Bendsøe and Kikuchi [19]. The enthusiasm for optimizing functional plate or shell structures never recedes [20,21]. Recent studies [22-25] demonstrate that topology optimization is a powerful tool to obtain high-Q designs. In this work, we numerically model the high-stress membrane structures via the finite element method and implement two simple numerical damping models to represent intrinsic and extrinsic damping loss, respectively. Density-based approaches are employed to formulate the design problem into a gradientbased topology optimization form. The optimization objective is the mechanical quality factor $Q$ or mechanical frequency-quality factor $Q f$ in the fundamental mode. The optimized results display significant improvements both on $Q$ and $Q f$ evaluated numerically, compared to the existing trampoline-like designs [16, 17,26]. Crucially, all the optimized designs exceed the minimum requirement for room-temperature quantum optomechanics, i.e. $6.2 \times 10^{12} \mathrm{~Hz}$ for $Q f$.

The remainder of the paper is organized as follows. In Section 2, the numerical approaches used to model prestressed membranes are briefly introduced. The formulation for topology optimization is presented in Section 3. Finally, design examples are presented and investigated in Section 4 before conclusions are drawn in Section 5.

\section{Modelling prestressed membranes}

\subsection{Prestressed structures}

To model prestressed membrane resonators, we use the primitive version of the 4-node MITC (Mixed Interpolation of Tensorial Components) quadrilateral shell element [27]. The constitutive relation for the shell elements follows a plane stress assumption that is customary in shell theory. Based on physical observation and numerical experiments, a two-step linear modeling process as suggested by Pedersen [20] is adequate. The first step focuses on a linear static analysis to establish the prestressed system, considering the element initial stress $\sigma_{0, e}$ by solving the equilibrium equation as 


$$
\begin{gathered}
\boldsymbol{K}_{0} \boldsymbol{U}_{0}=\boldsymbol{F}_{\sigma} \\
\boldsymbol{K}_{0}=\sum_{e} \boldsymbol{K}_{0, e} \quad, \quad \boldsymbol{K}_{0, e}=\int_{V_{0, e}} \boldsymbol{B}_{\mathrm{L} 0}^{\mathrm{T}} \boldsymbol{D}_{e} \boldsymbol{B}_{\mathrm{L} 0} \mathrm{~d} V \\
\boldsymbol{F}_{\sigma}=\sum_{e} \boldsymbol{F}_{\sigma, e} \quad, \quad \boldsymbol{F}_{\sigma, e}=-\int_{V_{0, e}} \boldsymbol{B}_{\mathrm{L} 0}^{\mathrm{T}} \boldsymbol{\sigma}_{0, e} \mathrm{~d} V
\end{gathered}
$$

where $\boldsymbol{B}_{\mathrm{L} 0}$ is the linear strain-displacement matrix; $\boldsymbol{F}_{\sigma}$ is the load vector; $\boldsymbol{K}_{0}$ is the linear stiffness matrix of the system and $\boldsymbol{D}_{e}$ is the element constitutive matrix.

The second step aims at investigating the dynamic characteristics of the high-prestress system by solving the corresponding eigenvalue problem as

$$
\begin{gathered}
\left(\boldsymbol{K}_{\mathrm{t}}+\mathrm{i} \boldsymbol{C}-\lambda_{j} \boldsymbol{M}\right) \boldsymbol{\phi}_{j}=\mathbf{0} \\
\lambda_{j}=\omega_{j}^{2}=\left(\omega_{\mathrm{r}, j}+\mathrm{i} \omega_{\mathrm{s}, j}\right)^{2} \\
f_{j}=\frac{\omega_{\mathrm{r}, j}}{2 \pi}, \quad Q_{j}=\frac{\omega_{\mathrm{r}, j}}{2 \omega_{\mathrm{s}, j}}, \quad(Q f)_{j}=Q_{j} \frac{\omega_{\mathrm{r}, j}}{2 \pi}
\end{gathered}
$$

where $\lambda_{j}$ is the $j$-th eigenvalue; $\omega_{j}$ is the $j$-th angular frequency which contains the real part $\omega_{\mathrm{r}, j}$ and the imaginary part $\omega_{j} ; f_{j}, Q_{j}$ and $(Q f)_{j}$ are the $j$-th frequency, quality factor and frequency-quality factor, respectively; $\boldsymbol{\phi}_{j}$ is the $j$-th eigenvector; $\boldsymbol{M}=\sum_{e} \boldsymbol{M}_{e}$ is the consistent mass matrix, using element mass density $\varsigma_{e}$ and shape function matrix $\boldsymbol{N}$ as $\boldsymbol{M}_{e}=\int_{V_{0, e}} \zeta_{e} \boldsymbol{N}^{\mathrm{T}} \boldsymbol{N} \mathrm{d} V=\varsigma_{e} \overline{\boldsymbol{M}}_{0} ; \boldsymbol{K}_{\mathrm{t}}=\sum_{e} \boldsymbol{K}_{\mathrm{t}, e}$ is the tangent stiffness matrix and the damping matrix $\boldsymbol{C}$ will be discussed later. Due to the assumption of small initial displacements, $\boldsymbol{K}_{\mathrm{t}, e}$ can be simplified by only including two parts as

$$
\boldsymbol{K}_{\mathrm{t}, e}=\int_{V_{0, e}} \boldsymbol{B}_{\mathrm{L} 0}^{\mathrm{T}} \boldsymbol{D}_{e} \boldsymbol{B}_{\mathrm{L} 0} \mathrm{~d} V+\int_{V_{0, e}} \boldsymbol{B}_{\mathrm{NL}}^{\mathrm{T}} \boldsymbol{\sigma}_{e} \boldsymbol{B}_{\mathrm{NL}} \mathrm{d} V=\boldsymbol{K}_{0, e}+\boldsymbol{K}_{\sigma, e}
$$

where $\boldsymbol{B}_{\mathrm{NL}}$ is the nonlinear strain-displacement matrix, cf. Table 6.5 in [28] and $\boldsymbol{\sigma}_{e}$ is the element stress. The second term of (3) is denoted as $\boldsymbol{K}_{\sigma, e}$, which is also called the "initial stress stiffness matrix" or "geometric stiffness matrix". To assemble $\boldsymbol{K}_{\sigma, e}$, the stress term $\boldsymbol{\sigma}_{e}$ in the integral is separated into two parts: the contribution from the element initial stress $\sigma_{0, e}$, and the contribution from the obtained displacement field $\boldsymbol{U}_{0}=\sum_{e} \boldsymbol{U}_{0, e}$. The stress term can be expressed by the combination of the current element strain $\boldsymbol{\varepsilon}_{e}$ and the element constitutive matrix $\boldsymbol{D}_{e}$ as

$$
\begin{gathered}
\boldsymbol{\sigma}_{e}=\boldsymbol{D}_{e} \varepsilon_{e}=\boldsymbol{D}_{e}\left(\boldsymbol{\varepsilon}_{0}+\boldsymbol{\varepsilon}_{1, e}\right)=E_{e} \overline{\boldsymbol{D}}\left(\varepsilon_{0}+\boldsymbol{B}_{\mathrm{L} 0} \boldsymbol{U}_{0, e}\right)=\boldsymbol{\sigma}_{0, e}+\boldsymbol{\sigma}_{1, e} \\
\boldsymbol{\sigma}_{1, e}=\boldsymbol{D}_{e} \boldsymbol{\varepsilon}_{1, e}
\end{gathered}
$$

where $\boldsymbol{\varepsilon}_{0}$ is the initial strain which corresponds to the element initial stress $\boldsymbol{\sigma}_{0, e}$ via $\boldsymbol{\sigma}_{0, e}=\boldsymbol{D}_{e} \boldsymbol{\varepsilon}_{0}$, and $\boldsymbol{\varepsilon}_{1, e}=$ $\boldsymbol{B}_{\mathrm{L} 0} \boldsymbol{U}_{0, e}$ is the element strain generated by the static analysis, cf. (1). To relate the element geometric stiffness matrix $\boldsymbol{K}_{\sigma, e}$ to design variables, the Young's modulus $E_{e}$ is separated from $\boldsymbol{D}_{e}$ as $\boldsymbol{D}_{e}=E_{e} \overline{\boldsymbol{D}}$. Further, $\boldsymbol{K}_{\sigma, e}$ is rewritten as the sum of two parts

$$
\boldsymbol{K}_{\sigma, e}=E_{e} \int_{V_{0, e}} \boldsymbol{B}_{\mathrm{NL}}^{\mathrm{T}}\left(\overline{\boldsymbol{D}} \boldsymbol{\varepsilon}_{0}\right) \boldsymbol{B}_{\mathrm{NL}} d V+E_{e} \int_{V_{0, e}} \boldsymbol{B}_{\mathrm{NL}}^{\mathrm{T}}\left(\overline{\boldsymbol{D}} \boldsymbol{B}_{\mathrm{L} 0} \boldsymbol{U}_{0, e}\right) \boldsymbol{B}_{\mathrm{NL}} d V
$$




$$
=\boldsymbol{K}_{\mathrm{IniS}, e}+\boldsymbol{K}_{\mathrm{Disp}, e}
$$

where $\boldsymbol{K}_{\text {Inis, } e}$ and $\boldsymbol{K}_{\text {Disp }, e}$ represent the contributions from $\boldsymbol{\sigma}_{0, e}$ and $\boldsymbol{U}_{0, e}$, respectively.

Since the eigenvalues of the prestressed membranes are calculated after the in-plane static analysis, the resulting $\boldsymbol{\sigma}_{e}$ at some regions may contain compressive components that cause instability in the eigenvalue analysis. The instability is featured by close-to-zero eigenvalues in (2), and wrinkling-like modal profiles. This instability is taken care of in the optimization process as described in Section 3.1.

\subsection{Damping consideration}

Damping is ubiquitous in realistic dynamic systems. Although characteristics like mass and stiffness are fairly easy to calculate, damping is considerably harder to estimate with any sort of certainty. In general, there are three main sources of damping in micro mechanical resonators [29]: (1) the interaction between a resonator and its surrounding medium, e.g. a viscous liquid or a rarefied ballistic gas; (2) boundary damping caused by the propagation of elastic waves to the substrate; and (3) intrinsic dissipation mechanisms. Since viscous interaction losses can be readily overcome by operation in vacuum, only boundary and intrinsic damping are taken into account in the following.

\subsubsection{Boundary damping}

The $\mathrm{Si}_{3} \mathrm{~N}_{4}$ membrane is commercially deposited upon and suspended from a relatively thick silicon wafer, also called the substrate. The suspended structure is held flat by the internal stress (nominally around $1 \mathrm{GPa}$ ). The behavior of mechanical resonators at low frequencies is strongly affected by the boundary conditions. It is not possible to provide ideal boundary conditions, i.e. simply supported or clamped, for realistic structures. In fact, the silicon substrate that acts as the support of the suspended structure is not rigid and shows flexibility to some degree. In addition, the substrate plays an important role in clamping losses for out-of-plane fundamental modes [17]. Due to high prestress, the rotation behavior is strongly suppressed along the support boundaries. Therefore, the boundaries of the suspended membrane structure are considered as elastic support edges and simultaneously provide boundary loss by vertical hysteretic damping, as shown in Fig. 2. The boundary conditions are expressed as

$$
\begin{gathered}
x=-l_{b}: \Psi_{x}=0, \Psi_{y}=0, \quad \bar{k}_{\mathrm{b}} w=-Q_{x} \\
x=l_{b}: \Psi_{x}=0, \Psi_{y}=0, \quad \bar{k}_{\mathrm{b}} w=Q_{x} \\
y=-l_{a}: \Psi_{x}=0, \Psi_{y}=0, \bar{k}_{\mathrm{b}} w=-Q_{y} \\
y=l_{a}: \Psi_{x}=0, \Psi_{y}=0, \bar{k}_{\mathrm{b}} w=Q_{y} \\
Q_{x}=\kappa G h\left(\Psi_{x}+\frac{\partial w}{\partial x}\right) \\
Q_{y}=\kappa G h\left(\Psi_{y}+\frac{\partial w}{\partial y}\right) \\
\bar{k}_{\mathrm{b}}=\left(1+\mathrm{i} \eta_{\mathrm{b}}\right) k_{\mathrm{b}}
\end{gathered}
$$

where $\Psi_{x}$ and $\Psi_{y}$ are the rotations along $x$-direction and $y$-direction, respectively; $Q_{x}$ and $Q_{y}$ are the transverse shearing forces; $h$ is the thickness of the membrane; $w$ is the deflection; $\kappa$ is the shear correction factor $(\kappa=5 / 6) ; G$ is the shear modulus; $\eta_{b}$ is the hysteretic damping coefficient; $k_{\mathrm{b}}$ is a constant representing the stiffness of elastic supports $\left(k_{\mathrm{b}}=8.315 \times 10^{7} \mathrm{kN} / \mathrm{m}^{2}\right)$. The imaginary part indicates that the damping force is proportional to the displacement amplitude but in phase with the velocity. 


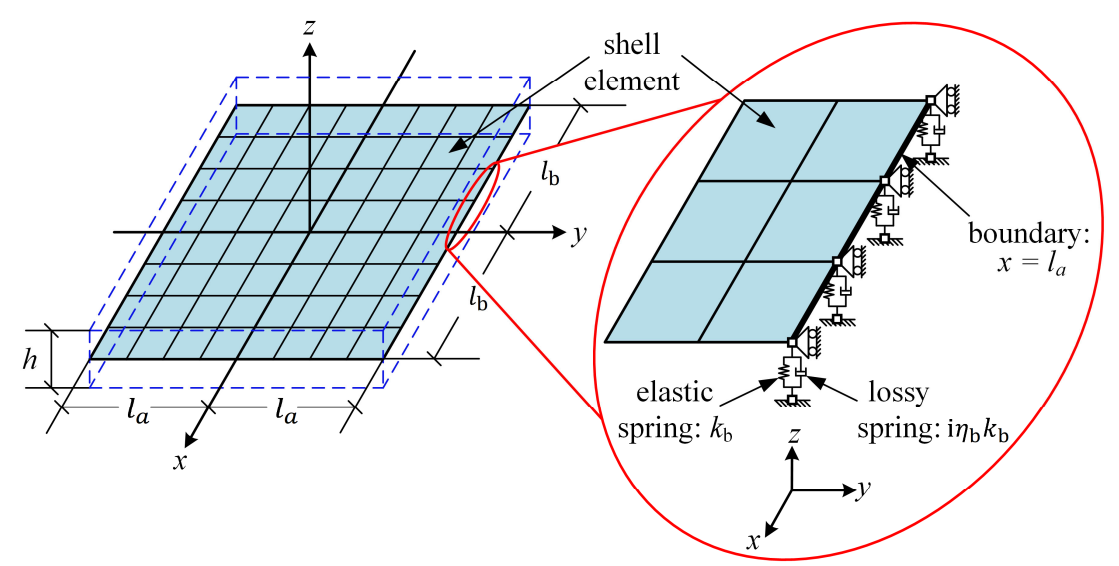

Fig. 2. Schematic of boundary conditions for the shell model including boundary damping. The special symbols for boundary condition indicate that boundaries are fixed in all degrees of freedom except the out of plane direction that is constrained by springs and dampers.

\subsubsection{Intrinsic damping}

The intrinsic damping is embedded into the eigenvalue problem, cf. (2), through a relaxation mechanism described by a complex-valued Young's modulus $\tilde{E}_{e}=\left(1+\mathrm{i} \eta_{\mathrm{s}}\right) E_{e}[12,13,30]$, where $\eta_{\mathrm{s}}$ is defined as the intrinsic loss factor. Due to the complex-valued Young's modulus, the element constitutive matrix $\boldsymbol{D}_{e}$ becomes a complex form as $\widetilde{\boldsymbol{D}}_{e}=\widetilde{E}_{e} \overline{\boldsymbol{D}}$. The imaginary part of $\widetilde{\boldsymbol{D}}_{e}$, i.e. $\eta_{\mathrm{s}} E_{e} \overline{\boldsymbol{D}}$, indicates that damping forces are proportional to material deformation during oscillations. The integral process as the first term in (3) yields the element intrinsic damping matrix $\boldsymbol{C}_{\mathrm{Intr}, e}$ as

$$
\boldsymbol{C}_{\mathrm{Intr}, e}=\int_{V_{0, e}} \boldsymbol{B}_{\mathrm{L} 0}^{\mathrm{T}}\left(\eta_{\mathrm{s}} E_{e} \overline{\boldsymbol{D}}\right) \boldsymbol{B}_{\mathrm{L} 0} \mathrm{~d} V=\eta_{\mathrm{s}} \boldsymbol{K}_{0, e}
$$

Since the element stress $\boldsymbol{\sigma}_{e}$ in (3) results from the static analysis, cf. (1), $\boldsymbol{\sigma}_{e}$ is viewed as constant in the eigenvalue analysis, cf. (2). Unlike $\boldsymbol{C}_{\mathrm{Intr}, e}, \boldsymbol{K}_{\sigma, e}$ has nothing to do with material deformation during oscillations. Hence, the imaginary part of $\widetilde{\boldsymbol{D}}_{e}$ never enters (5) when performing interpolations described in Section 3. Since the established intrinsic damping is independent of $\boldsymbol{K}_{\sigma}$ generated by prestress, the quality factor of a structure rises when prestress increases. This adheres to the relationship between prestress and $Q$ reported in the literature.

To consider the two damping sources simultaneously, (2) should be supplemented by corresponding damping terms. The tangent stiffness matrix $\boldsymbol{K}_{\mathrm{t}}$ in (2) needs to include $\boldsymbol{K}_{\mathrm{B}}$ as $\boldsymbol{K}_{\mathrm{t}}=\boldsymbol{K}_{\mathrm{B}}+\boldsymbol{K}_{0}+\boldsymbol{K}_{\sigma}$ compared to (3), assuming that $\boldsymbol{K}_{\mathrm{B}}$ contains the stiffness contribution from the elastic supports described by $k_{\mathrm{b}}$ in (6). Also, the damping matrix $\boldsymbol{C}$ should combine the boundary damping from $\eta_{\mathrm{b}} k_{\mathrm{b}}$ and the intrinsic damping from $\eta_{s} K_{0}$.

\subsubsection{Calibration}

Since the weight between boundary damping and intrinsic damping is unknown, the values of damping coefficients $\eta_{\mathrm{b}}$ and $\eta_{\mathrm{s}}$ are determined through a calibration process trying to mimic the experimental data of the reference design reported by Norte et al. [17], see Fig. 3. The trampoline-like reference design is implemented as a numerical model based on shell elements. The membrane thickness is $50 \mathrm{~nm}$. The material parameters include Young's modulus of $250 \mathrm{GPa}$, Poisson ratio of 0.23 , and mass density $\varsigma_{0}$ of $3100 \mathrm{~kg} / \mathrm{m}^{3}$, which corresponds to $\mathrm{Si}_{3} \mathrm{~N}_{4}$. The initial stress of $1 \mathrm{GPa}$ for biaxial tension is achieved by using a constant $\varepsilon_{0}$ $=0.00308$, cf. (4), in each element. As the specified geometry of the trampoline-like reference design, the 
edges of the black areas connected to the red lines in Fig. 3 are interpreted as boundaries and support the resonator from the four corners. The boundary conditions refer to Fig. 2 and (6).

In the calibration process, the trampoline-like reference design is numerically evaluated with different combinations of $\eta_{\mathrm{b}}$ and $\eta_{\mathrm{s}}$ until the $Q$ value is close to the reference $Q=17.16 \times 10^{6}$. In this way, five cases are established to reflect a transition of the dominant damping source from intrinsic damping to boundary damping. We define that Case 1 only includes intrinsic damping, i.e. where $\eta_{\mathrm{b}}=0$, whereas Case 5 only includes boundary damping, i.e. where $\eta_{\mathrm{s}}=0$. Other information on $\eta_{\mathrm{b}}$ and $\eta_{\mathrm{s}}$ for each case is listed in Table 1. The calibration results are illustrated in Fig. 4 where the curves are smoothed by including more sampling points from a cubic spline interpolation on the damping coefficients listed in Table 1 at a fixed interval equal to $1 / 20$ of the $\eta_{\mathrm{b}}$ in Case 5 . The transition of the intrinsic damping proportion from Case 1 to Case 5 follows a nearly linear decline.

Table 1 Damping coefficients in each case.

\begin{tabular}{cccc}
\hline Case & $\eta_{\mathrm{s}}\left[\times 10^{-4}\right]$ & $\eta_{\mathrm{b}}$ & $Q\left[\times 10^{6}\right]$ \\
\hline 1 & 2.513 & 0.000 & 17.1769 \\
2 & 1.801 & 0.095 & 17.1739 \\
3 & 1.140 & 0.190 & 17.1507 \\
4 & 0.518 & 0.285 & 17.1488 \\
5 & 0.000 & 0.380 & 17.1371 \\
\hline
\end{tabular}

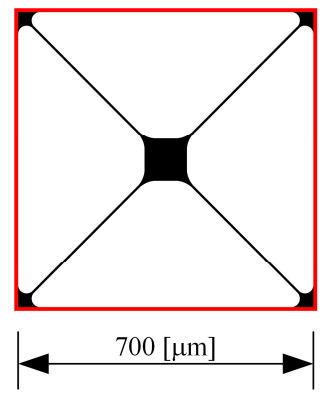

$$
\begin{aligned}
f & =172 \mathrm{kHz} \\
Q & =17.16 \times 10^{6} \\
Q f & =2.9 \times 10^{12} \mathrm{~Hz}
\end{aligned}
$$

Fig. 3. The trampoline-like reference design suggested by Norte et al. [17]. Red lines indicate the boundary conditions specified in Fig. 2.

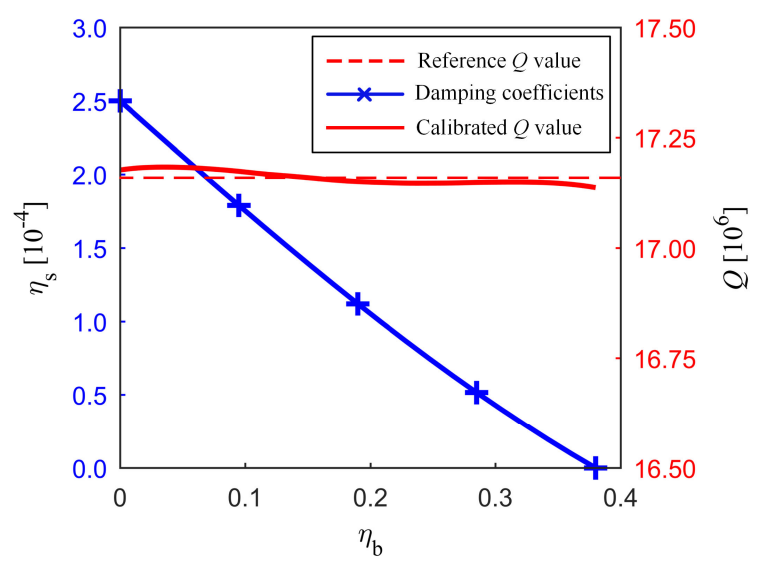

Fig. 4. Calibration results of the reference design.

To compare with the experimental data in figure 3 from Norte et al. [17], we sweep the membrane thickness in numerical experiments under different damping combinations, from Case 1 (pure intrinsic damping) to Case 5 (only boundary damping). The relationship between $Q f$ s and thicknesses is plotted in Fig. 
5. The $Q f$ s experience a significant increase when the membrane becomes thinner in all cases. The advantage of thinner membranes shows insensitivity to the weight between intrinsic damping and boundary damping, though $Q f$ s show the fastest growth in Case 1, according to the zoom-in parts in Fig. 5.

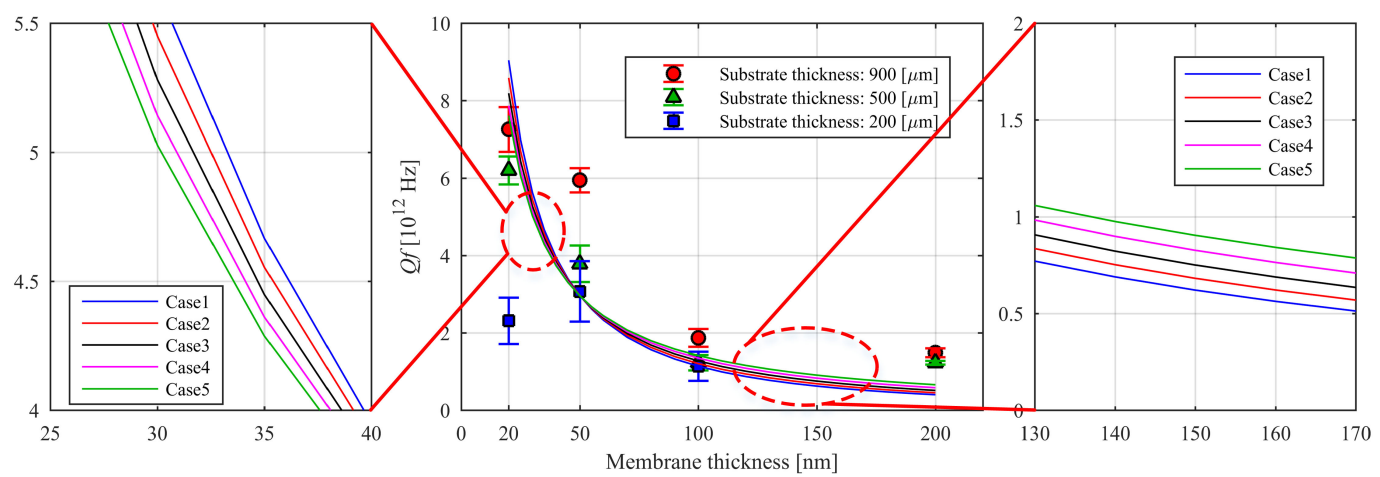

Fig. 5. Frequency-quality factor as a function of membrane thickness in numerical experiments. The experimental data comes from figure 3 in Norte et al. [17].

\section{Topology optimization}

\subsection{Interpolation scheme}

A "density-based topology optimization approach", which followed the groundbreaking studies [31,32] and became an efficient form in [33,34], is employed to turn a 0-1 distribution problem into a continuous distribution problem. A design variable $\rho_{e}$ is assigned to each element. The Young's modulus $E_{e}$ of element $e$ is directly related to the projected physical density $\bar{\rho}_{e}$ according to the Rational Approximation of Material Properties (RAMP) model [35] as

$$
E_{e}=E_{\min }+\frac{\bar{\rho}_{e}}{1+q\left(1-\bar{\rho}_{e}\right)}\left(E_{0}-E_{\min }\right), \quad \bar{\rho}_{e} \in[0,1]
$$

where $q \geq 0$ is the penalization parameter; $E_{0}$ is the Young's modulus; usually $E_{\min }$ is a very small value assigned to void regions in order to prevent the global stiffness matrix from becoming singular; $\bar{\rho}_{e}$ is the projected physical density [36] calculated by

$$
\bar{\rho}_{e}=\frac{\tanh \left(\beta_{\mathrm{f}} \eta\right)+\tanh \left(\beta_{\mathrm{f}}\left(\tilde{\rho}_{i}-\eta\right)\right)}{\tanh \left(\beta_{\mathrm{f}} \eta\right)+\tanh \left(\beta_{\mathrm{f}}(1-\eta)\right)}
$$

where $\eta$ and $\beta_{\mathrm{f}}$ control the shape of (9) by gradually increasing $\beta_{\mathrm{f}}$ through the optimization process; $\tilde{\rho}_{e}$ is the filtered density of element $e$, which is calculated as

$$
\tilde{\rho}_{e}=\frac{1}{\sum_{i \in n_{e}} H_{e, i}} \sum_{i \in n_{e}}\left(\rho_{i} H_{e, i}\right)
$$

where $n_{e}$ is the set of element $i$ for which the center-to-center distance $\Delta(e, i)$ to element $e$ is smaller than the filter radius $r_{\min }$, and $H_{e, i}$ is a weight factor defined as $H_{e, i}=\max \left(0, r_{\min }-\Delta(e, i)\right)$. Due to the RAMP scheme, $\boldsymbol{D}_{e}, \boldsymbol{K}_{0, e}, \boldsymbol{K}_{\sigma, e}$ and $\boldsymbol{F}_{\sigma, e}$ in Section 2.1 are connected to design variables. In contrast, the element volume $V_{e}$ and mass density $\zeta_{e}$ follow linear interpolations as $V_{e}=\bar{\rho}_{e} V_{0}$ and $\zeta_{e}=\zeta_{\min }+\left(\zeta_{0}-\zeta_{\min }\right) \bar{\rho}_{e}$, respectively, where $V_{0}$ represents the volume of a shell element and $\varsigma_{0}$ is the mass density of material. 
The structural layout design towards maximizing natural frequencies has been studied for more than two decades, by e.g. [20,37-41]. One basic problem in the topology optimization of eigenvalues using elementdensity based approaches is the occurrence of spurious modes, see [20,39] for details. These spurious modes are caused by inappropriate stiffness-to-mass ratio in low-density regions, where element densities are close to the minimum value. Since element stiffness is lower than element mass, the stiffness-to-mass ratio of these elements becomes extremely low compared to solid parts. Therefore, these low-density elements or regions may control the lowest eigenmodes of the whole structure. One possible strategy for suppressing spurious modes is raising the stiffness-to-mass ratio of low-density elements by modifying the interpolation scheme used for stiffness and mass $[20,39]$. This is achieved by setting $E_{\min }=10^{-6} E_{0}$ and $\varsigma_{\min }=10^{-7} \varsigma_{0}$ in this study.

For low-density elements, $\boldsymbol{\sigma}_{e}$ resulting from the in-plane static analysis, cf. (1), may cause wrinklinglike instabilities in the eigenvalue analysis due to its compressive components. Adequately suppressing $\boldsymbol{\sigma}_{1, e}$ $=\boldsymbol{D} \boldsymbol{B}_{\mathrm{L} 0} \boldsymbol{U}_{0, e}$ in low-density regions can make sure $\boldsymbol{\sigma}_{e}$ remains in biaxial tension. Therefore, an interpolated $\widetilde{\boldsymbol{U}}_{0, e}$ inspired by the literature [42] instead of $\boldsymbol{U}_{0, e}$ is employed as

$$
\begin{gathered}
\widetilde{\boldsymbol{U}}_{0, e}=\alpha_{e} \boldsymbol{U}_{0, e}, \quad \boldsymbol{\sigma}_{1, e}=\boldsymbol{D} \boldsymbol{B}_{\mathrm{L} 0} \widetilde{\boldsymbol{U}}_{0, e} \\
\alpha_{e}=\frac{\tanh (\chi \Upsilon)+\tanh \left(\chi\left(\frac{E_{e}}{E_{0}}-\Upsilon\right)\right)}{\tanh (\chi \Upsilon)+\tanh (\chi(1-\Upsilon))}
\end{gathered}
$$

where $\alpha_{e}$ is the scaling factor; $\gamma$ and $\chi$ are constant parameters for tuning. In this study, $\chi=30$ and $\Upsilon=0.05$. The displacement interpolation scheme (11) aims at suppressing $\sigma_{1, e}$ for the gray elements whose Young's modulus are smaller than $\Upsilon E_{0}$ in order to avoid instabilities in the eigenvalue analysis. Also note that, $\widetilde{\boldsymbol{U}}_{0, e}$ is equal to $\boldsymbol{U}_{0, e}$ for solid elements.

\subsection{Robust formulation}

To promote length-scale control, robustness to manufacturing variations and nearly binary designs, the objective function is transformed into a max-min form via the combination of three different realizations together following the robust formulation suggested by Wang et al. [36] where the intermediate design can be viewed as the blue-print design. The basic requirement of the robust formulation is to maximize the minimum $Q$ or $Q f$ among three realizations, corresponding to the eroded, the intermediate (blue-print) and the dilated designs. These three structures are represented by one set of design variables $\boldsymbol{\rho}$ but with three different thresholds, i.e. $\eta_{\mathrm{E}}, \eta_{\mathrm{I}}$ and $\eta_{\mathrm{D}}$ for the eroded, intermediate and dilated design, respectively, in the projection process (9). Its mathematical description is as follows.

$$
\begin{aligned}
& \max _{\boldsymbol{\rho}}\left(\min \left(\ln \left(Q\left(\overline{\boldsymbol{\rho}}_{p}\right)\right) \mid p \in\left\{\eta_{\mathrm{E}}, \eta_{\mathrm{I}}, \eta_{\mathrm{D}}\right\}\right)\right) \\
& \text { or } \\
& \max _{\boldsymbol{\rho}}\left(\min \left(\ln \left(Q f\left(\overline{\boldsymbol{\rho}}_{p}\right)\right) \mid p \in\left\{\eta_{\mathrm{E}}, \eta_{\mathrm{I}}, \eta_{\mathrm{D}}\right\}\right)\right) \\
& \text { s.t. : } \\
& \boldsymbol{K}_{0}\left(\overline{\boldsymbol{\rho}}_{p}\right) \boldsymbol{U}_{0}=\boldsymbol{F}_{\sigma}\left(\overline{\boldsymbol{\rho}}_{p}\right), \quad p \in\left\{\eta_{\mathrm{E}}, \eta_{\mathrm{I}}, \eta_{\mathrm{D}}\right\} \\
& 1-\frac{\omega_{\mathrm{r}, 1}\left(\overline{\boldsymbol{\rho}}_{p}\right)}{\omega_{\mathrm{L}}} \leq 0, \quad p \in\left\{\eta_{\mathrm{E}}, \eta_{\mathrm{I}}, \eta_{\mathrm{D}}\right\}
\end{aligned}
$$




$$
\begin{aligned}
& \frac{1}{\Gamma_{\mathrm{V}}} \frac{\sum_{e} V_{e}\left(\overline{\boldsymbol{\rho}}_{\eta_{\mathrm{D}}}\right)}{\sum_{e} V_{0}}-1 \leq 0 \\
& 0 \leq \rho_{e} \leq 1
\end{aligned}
$$

where $\overline{\boldsymbol{\rho}}_{\eta_{\mathrm{E}}}, \overline{\boldsymbol{\rho}}_{\eta_{\mathrm{I}}}$ and $\overline{\boldsymbol{\rho}}_{\eta_{\mathrm{D}}}$ can be calculated via (9) and (10); $\Gamma_{\mathrm{V}} \in(0,1)$ is the volume fraction limit; $\omega_{\mathrm{L}}$ is the frequency lower bound prescribed for the three realizations. The three realizations are based on the same filtered density $\widetilde{\boldsymbol{\rho}}$, and the dilated structure always has the highest volume. Hence, the volume constraint is only imposed on the dilated design. For a pure mathematical optimization problem of maximizing $Q$ or $Q f$, neither volume constraint nor frequency constraint is necessary. However, for physical experiments the service frequency of the laser beam used to excite the resonators falls into a fixed range. Usually, it should be higher than $100 \mathrm{kHz}$. Also, a very fragile or disconnected structure could be useless for fabrication. Unfortunately, numerical experiments indicate that a very fragile structure that is almost disconnected to boundaries has an extremely high theoretical $Q$ or $Q f$. The frequency constraints act as an implicit method to provide a reasonable stiffness lower bound avoiding fragile or disconnected designs and ensuring practically relevant frequencies.

\subsection{Sensitivity analysis}

The derivative of a specific eigenvalue $\lambda_{j}$ with respect to a projected physical density $\bar{\rho}_{e}$, see the Appendix for detailed derivations, can be expressed as

$$
\begin{aligned}
\frac{\mathrm{d} \lambda_{j}}{\mathrm{~d} \bar{\rho}_{i}} & =\frac{\boldsymbol{\phi}_{j}^{\mathrm{T}}\left(\frac{\mathrm{d} \boldsymbol{K}_{0}}{\mathrm{~d} \bar{\rho}_{i}}+i \frac{\mathrm{d} \boldsymbol{C}}{\mathrm{d} \bar{\rho}_{i}}+\frac{\partial \boldsymbol{K}_{\sigma}}{\partial \bar{\rho}_{i}}-\lambda_{j} \frac{\mathrm{d} \boldsymbol{M}}{\mathrm{d} \bar{\rho}_{i}}\right) \boldsymbol{\phi}_{j}+\boldsymbol{\beta}^{\mathrm{T}}\left(\frac{\mathrm{d} \boldsymbol{F}_{\sigma}}{\mathrm{d} \bar{\rho}_{i}}-\frac{\mathrm{d} \boldsymbol{K}_{0}}{\mathrm{~d} \bar{\rho}_{i}} \boldsymbol{U}_{0}\right)}{\boldsymbol{\phi}_{j}^{\mathrm{T}} \boldsymbol{M} \boldsymbol{\phi}_{j}} \\
\frac{\mathrm{d} \boldsymbol{C}}{\mathrm{d} \bar{\rho}_{i}} & =\eta_{\mathrm{s}} \frac{\mathrm{d} \boldsymbol{K}_{0}}{\mathrm{~d} \bar{\rho}_{i}} \\
\frac{\partial \boldsymbol{K}_{\sigma}}{\partial \bar{\rho}_{i}} & =\frac{\mathrm{d} \boldsymbol{K}_{\text {Inis }}}{\mathrm{d} \bar{\rho}_{i}}+\frac{\partial \boldsymbol{K}_{\text {Disp }}}{\partial \alpha_{i}} \frac{\mathrm{d} \alpha_{i}}{\mathrm{~d} \bar{\rho}_{i}}+\frac{\partial \boldsymbol{K}_{\text {Disp }}}{\partial E_{i}} \frac{\mathrm{d} E_{i}}{\mathrm{~d} \bar{\rho}_{i}}
\end{aligned}
$$

where $\boldsymbol{\beta}$ is the adjoint vector that can be calculated through solving the following adjoint equation

$$
\boldsymbol{K}_{0}^{\mathrm{T}} \boldsymbol{\beta}=\left(\frac{\partial\left(\boldsymbol{K}_{\sigma} \boldsymbol{\phi}_{j}\right)}{\partial \boldsymbol{U}_{0}}\right)^{\mathrm{T}} \boldsymbol{\phi}_{j}
$$

The derivative of the specific eigenvalue $\lambda_{j}$ with respect to a design variable $\rho_{e}$ is achieved according to the chain rule [43]

$$
\frac{\mathrm{d} \lambda_{j}}{\mathrm{~d} \rho_{e}}=\sum_{i \in n_{e}}\left(\frac{\mathrm{d} \lambda_{j}}{\mathrm{~d} \bar{\rho}_{i}} \frac{\mathrm{d} \bar{\rho}_{i}}{\mathrm{~d} \tilde{\rho}_{i}} \frac{\mathrm{d} \tilde{\rho}_{i}}{\mathrm{~d} \rho_{e}}\right)
$$

where the definition of $n_{e}$ refers to Section 3.1. Further, the derivatives of the objective function, $\ln \left(Q_{j}\right)$ or $\ln \left(Q f_{j}\right)$, with respect to design variables are

$$
\begin{aligned}
& \frac{\mathrm{d}\left(\ln \left(Q_{j}\right)\right)}{\mathrm{d} \rho_{e}}=\frac{1}{\omega_{\mathrm{r}, j}} \frac{\mathrm{d} \omega_{\mathrm{r}, j}}{\mathrm{~d} \rho_{e}}-\frac{1}{\omega_{\mathrm{s}, j}} \frac{\mathrm{d} \omega_{\mathrm{s}, j}}{\mathrm{~d} \rho_{e}} \\
& \frac{\mathrm{d}\left(\ln \left(Q f_{j}\right)\right)}{\mathrm{d} \rho_{e}}=\frac{2}{\omega_{\mathrm{r}, j}} \frac{\mathrm{d} \omega_{\mathrm{r}, j}}{\mathrm{~d} \rho_{e}}-\frac{1}{\omega_{\mathrm{s}, j}} \frac{\mathrm{d} \omega_{\mathrm{s}, j}}{\mathrm{~d} \rho_{e}}
\end{aligned}
$$


where $\mathrm{d} \omega_{\mathrm{r}, j} / \mathrm{d} \rho_{e}, \mathrm{~d} \omega_{\mathrm{s}, j} / \mathrm{d} \rho_{e}$ can be derived from (2), see detailed expressions in the Appendix. Based on observations during the optimization process, the fundamental mode never experiences multiple eigenvalues. Techniques targeting multiple eigenvalues are hence unnecessary for this study.

\section{Numerical examples: maximizing $Q$ and $Q f$}

As shown in Fig. 6, a square design domain with a side length of $700 \mu \mathrm{m}$ and a thickness of $50 \mathrm{~nm}$ has a square passive region with a side length of $100 \mu \mathrm{m}$ at the center. This passive region is filled with solid elements. Utilizing symmetry of the square shape, a mesh of $200 \times 200$ square shell elements is used to discretize a quarter of the design domain. Consequently, this simplification retains the symmetric modes of the original structure but excludes the asymmetric ones. In a post-processing step, it has been verified that the fundamental mode of resulting designs is always a symmetric mode. The material parameters include Young's modulus of $250 \mathrm{GPa}$, Poisson ratio of 0.23 , and mass density $\varsigma_{0}$ of $3100 \mathrm{~kg} / \mathrm{m}^{3}$, which corresponds to $\mathrm{Si}_{3} \mathrm{~N}_{4}$ extensively used to fabricate membrane resonators in physical experiments. The initial stress of 1 GPa for biaxial tension is achieved by using a constant $\varepsilon_{0}=0.00308$, cf. (4), in each element. The design domain in Fig. 6 is consistent with the reference design in Fig. 3. The boundaries of the design domain are indicated by the red lines in Fig. 6. The boundary conditions refer to Fig. 2 and (6). The optimizations are performed under different damping combinations. The values of damping coefficients $\eta_{\mathrm{b}}$ and $\eta_{\mathrm{s}}$ follow the data listed in Table 1.

The method of moving asymptotes (MMA) introduced by Svanberg [44] plays the role of the optimizer to drive the optimization process. The value of $\beta_{\mathrm{f}}$ gradually goes up to a maximum value of 120 . The filter radius $r_{\min }$ spans 9 elements. The values of $\eta_{\mathrm{E}}, \eta_{\mathrm{I}}$ and $\eta_{\mathrm{D}}$ are $0.55,0.50$ and 0.45 , respectively. The volume fraction limit $\Gamma_{\mathrm{V}}$ is 0.4 , and the frequency lower bound $\omega_{\mathrm{L}}$ is $240 \mathrm{kHz}$. The penalization parameter $q$ in (8) is 3. Cases 1,3 and 5 start optimizations from a uniform density field, whereas Cases 2 and Case 4 employ the optimized results from Case 3 and Case 5 as initial guesses, respectively.

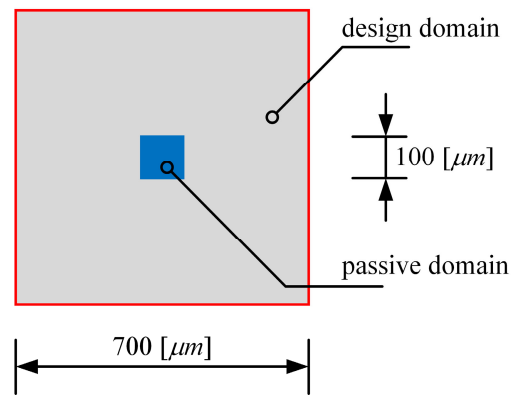

Fig. 6. Schematic of the design domain in optimizations. Red lines indicate the boundary conditions specified in Fig. 2.

The design objectives of the optimized results listed in Tables 2 and 3 correspond to maximizing $Q$ and $Q f$, respectively. Although their design objectives are different, in all cases the optimization targeting $Q$ yields similar structures as those targeting $Q f$ except for Case 4. The damping combinations cover the range from pure intrinsic loss (Case 1) to pure boundary loss (Case 5), providing a deeper understanding of the geometry transition following varying damping coefficients. In all cases, the feature that a suspended central pad well connected to the perimeter via four tethers can be easily identified and is similar to the reference design. Nevertheless, the four tethers gradually weaken their connection to the central pad from Case 1 to Case 5 . Since more material concentrates around the passive domain in Case 1, it looks like that the geometry of the central pad significantly extends from a prescribed side length of $100 \mu \mathrm{m}$ to $133 \mu \mathrm{m}$. In contrast, the central pads in other cases do not experience an extension in geometric dimensions. 
Converged designs rarely reach the volume fraction limit $\Gamma_{\mathrm{V}}=0.4$. Whereas all the eroded designs strictly cling to the frequency lower bound $\omega_{\mathrm{L}}=240 \mathrm{kHz}$. Due to the frequency lower bound, the optimized structures are well connected without any disconnected features that experience a non-physical oscillation with extremely high $Q$ when boundary damping dominates. Thanks to the frequency lower bound, the optimizations maximizing $Q$ not only enhance $Q$, but also improve $Q f$. As a result, the $Q f$ values of all the optimized designs circumvent a well-known trade-off between frequency and quality factor to exceed the minimum requirement for room-temperature quantum optomechanics, i.e. $6.2 \times 10^{12} \mathrm{~Hz}$. The optimization for the intermediate design accesses its most pronounced efficiency in Case 5, achieving $Q=66 \times 10^{6}$ and $Q f$ $=17.7 \times 10^{12} \mathrm{~Hz}$ that is around $280 \%$ and $510 \%$ higher than the corresponding values in the reference design. This indicates that topology optimization can intensively enhance the $Q$ and $Q f$ in a system which is dominated by boundary damping. The topological adaption to different damping conditions is especially pronounced at the boundaries. The boundary details are illustrated with enlarged insets in Tables 2 and 3. Studying these details reveals that as damping goes from pure intrinsic to pure boundary damping, the support topologies go from simple point supports to fully connected solid supports with slits between structure and support gradually disappearing in between. Note here that red lines indicate the external damped support as illustrated in Fig. 2, whereas all other boundaries are free. These observations confirm our intuition. Pure intrinsic damping does not care about boundary losses and hence much of the detrimental bending induced damping can be concentrated and isolated in small anchoring points to the substrate. Oppositely, pure boundary loss requires fully distributed boundaries to minimize the reaction forces and associated boundary damping. 
Table 2 Optimized structures for maximizing $Q$.

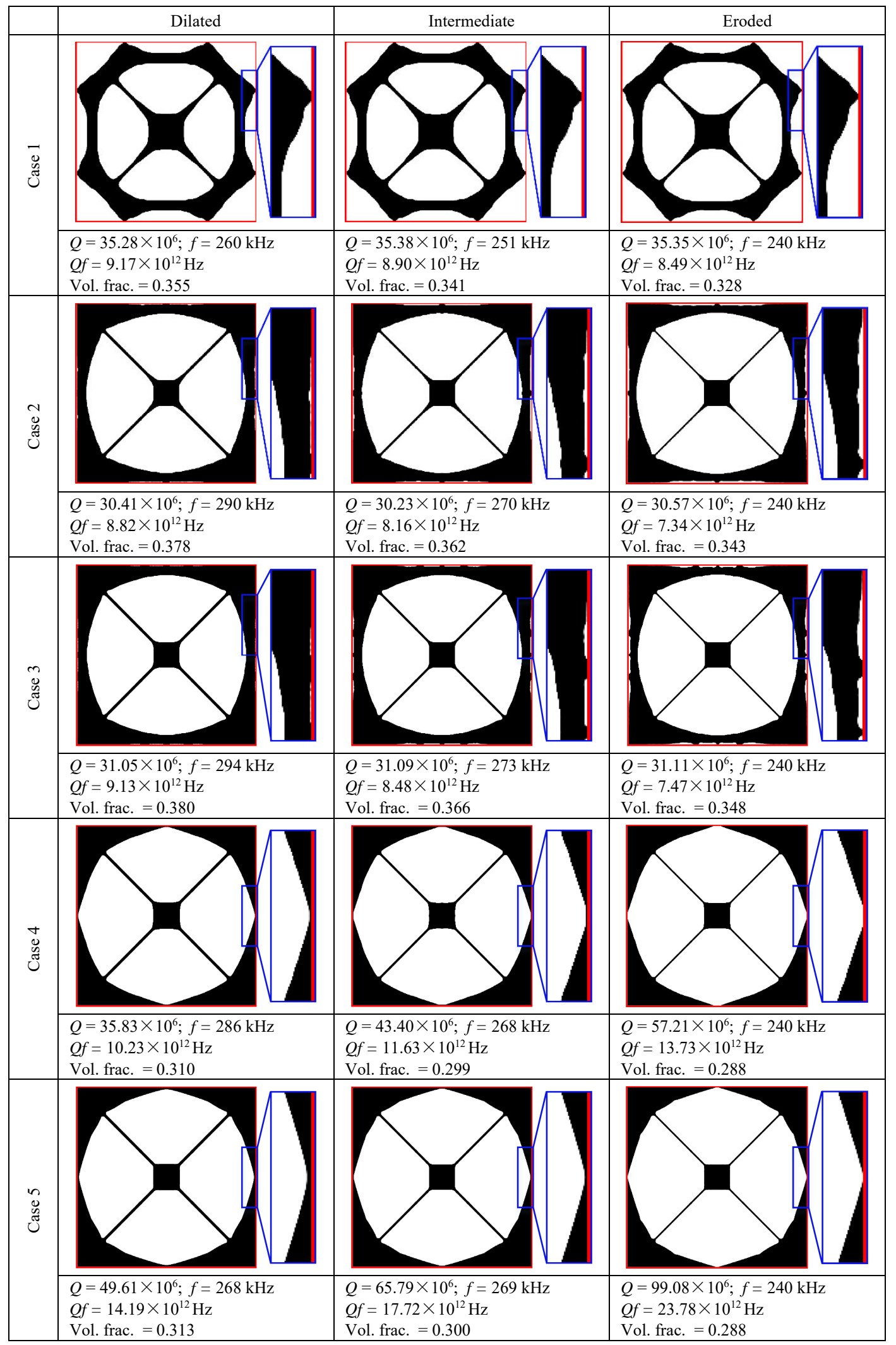


Table 3 Optimized structures for maximizing $Q f$.

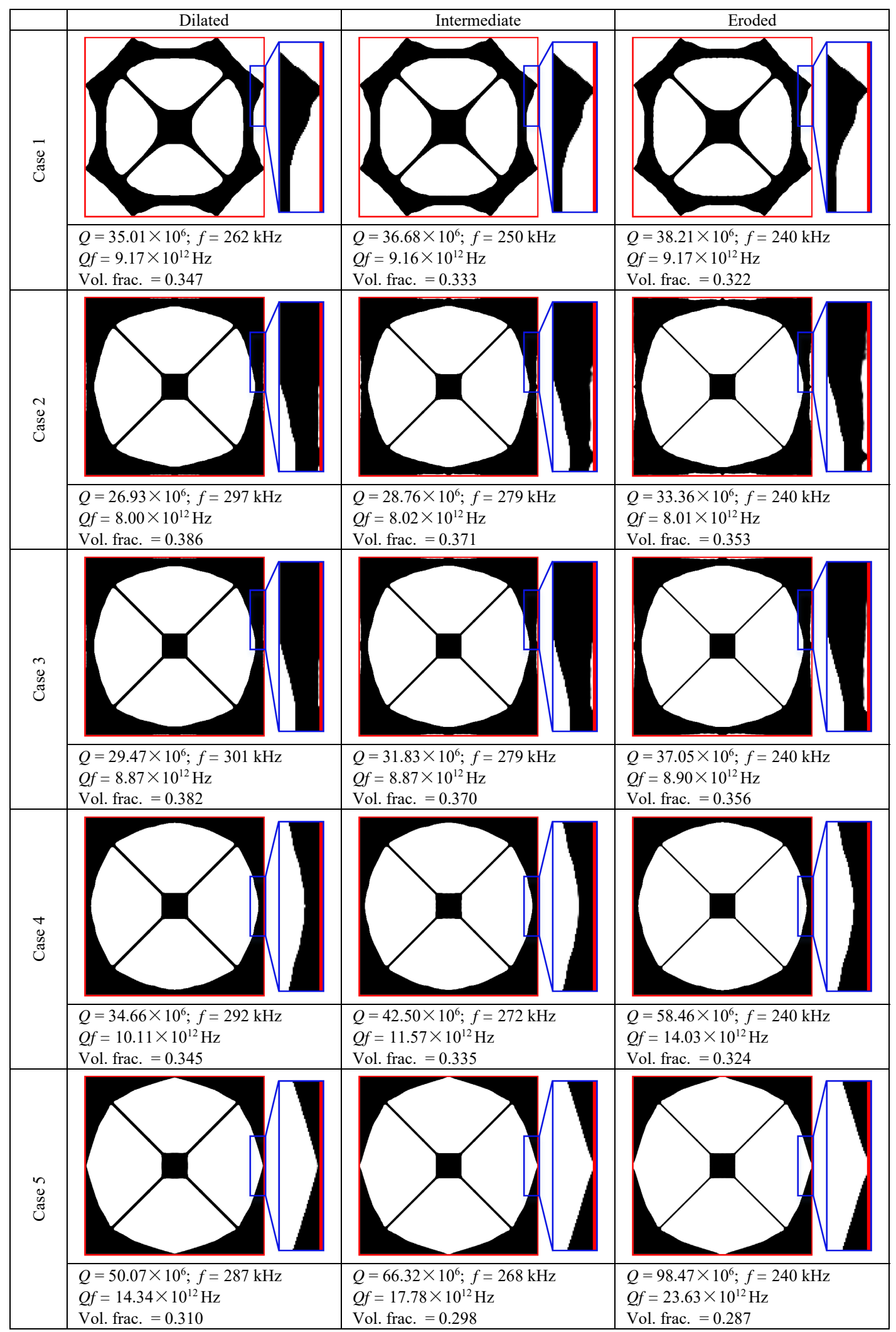


Usually, it is extremely hard to accurately estimate the proportion of intrinsic damping to boundary damping in real physical experiments. Due to this uncertainty, fabricated structures seldomly work in the same conditions as described in numerical simulations. It could be interesting to have a glimpse of the influence of this uncertainty. The performance of optimized results under different damping combinations is investigated and shown in Fig. 7 and Fig. 8. The optimized results (in Case 1) only targeting intrinsic damping are very sensitive to boundary damping. Their $Q$ or $Q f$ values descend rapidly once slight boundary damping enters their systems. Conversely, the optimizations under pure boundary damping yield designs that always experience a lower $Q$ and $Q f$ in a system where intrinsic damping is more pronounced. Unsurprisingly, the varying damping combinations exert less influence on the optimized designs arising from Case 3 in which intrinsic damping is on par with boundary damping. It demonstrates that simultaneously considering intrinsic and boundary damping can make the optimized designs balance the two damping sources well. Nevertheless, all the optimized designs beat others in their own cases, though their performance is impaired as damping conditions change. Furthermore, the performance gap between the three realizations reduces in a system only with pure intrinsic damping.

All the optimization processes are terminated once the continuation parameter $\beta_{\mathrm{f}}$ reaches 120 . Although the bulk of gray elements has been forced into a solid or void state at $\beta_{\mathrm{f}}=120$, there are still a few gray elements left in the design domain. Raising $\beta_{\mathrm{f}}$ to an extremely high value could be followed by unacceptable time cost, as well as numerical problems caused by high nonlinearities described by (9). As a post-processing verification approach, all the optimized designs are re-evaluated at $\beta_{\mathrm{f}}=300$ to investigate the influence of these remaining slightly gray elements, since manufacturing only realizes binary designs that exclude any gray regions. The re-evaluation results are shown in Fig. 7(b) and (d) and Fig. 8(b) and (d). A fluctuation can be observed according to the comparison of the curves of $Q$ (or $Q f$ ) at $\beta_{\mathrm{f}}=120$ and 300 . This demonstrates that $Q$ or $Q f$ really is quite sensitive to slight structural geometry variations. However, none of the optimized designs suffer from sudden decays in the $Q$ (or $Q f$ ) curves after post-processing. The robust formulation brings out designs that are insensitive to remaining gray elements. Therefore, the optimized designs are qualified as promising candidates for fabrication.

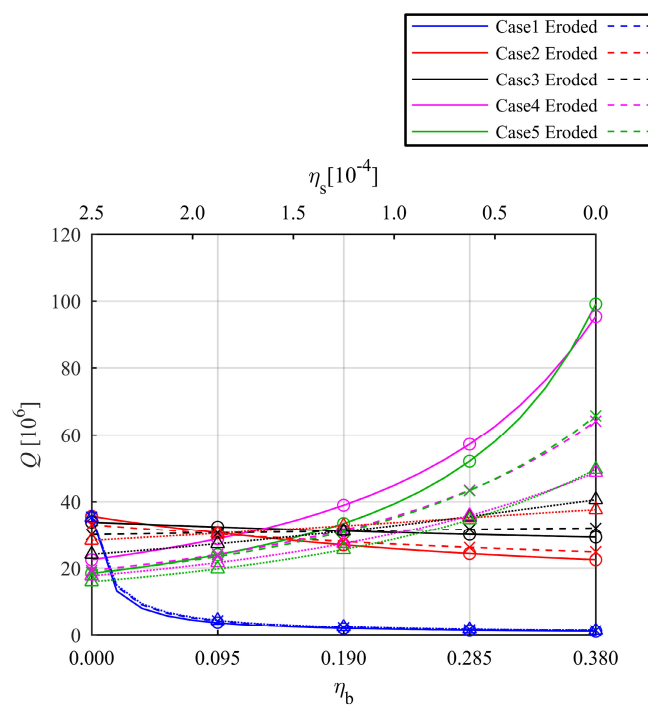

(a) $\eta_{\mathrm{s}}\left[10^{-4}\right]$

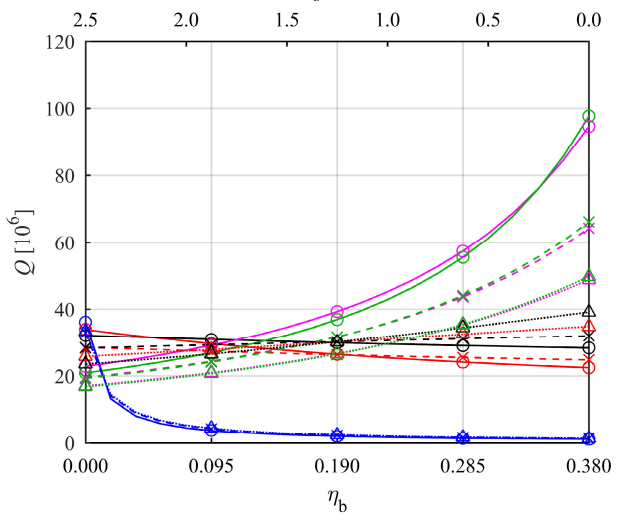

(b) 


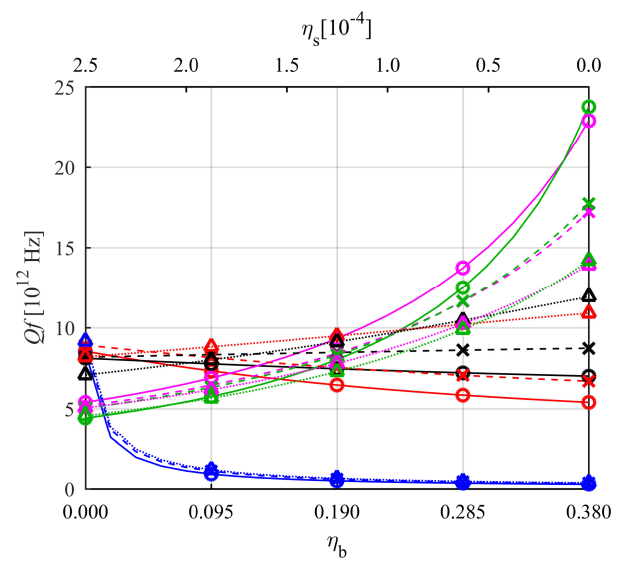

(c)

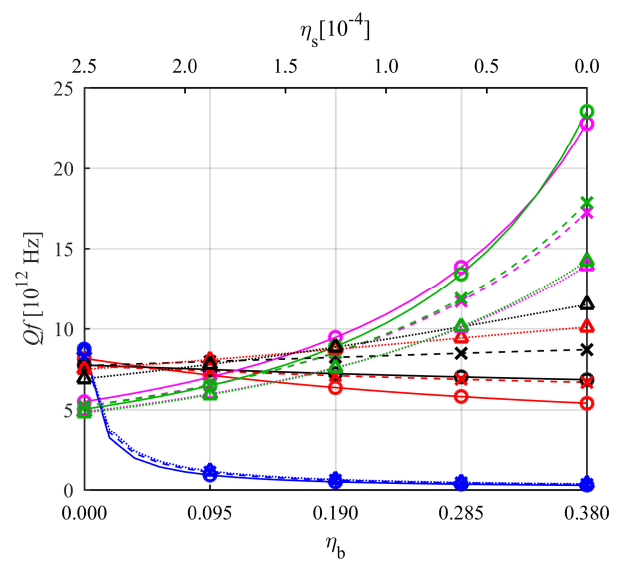

(d)

Fig. 7. Performance of maximizing- $Q$ designs under different damping combinations: (a) and (c) at $\beta_{\mathrm{f}}=$ 120 without post-processing; (b) and (d) re-evaluations at $\beta_{\mathrm{f}}=300$.

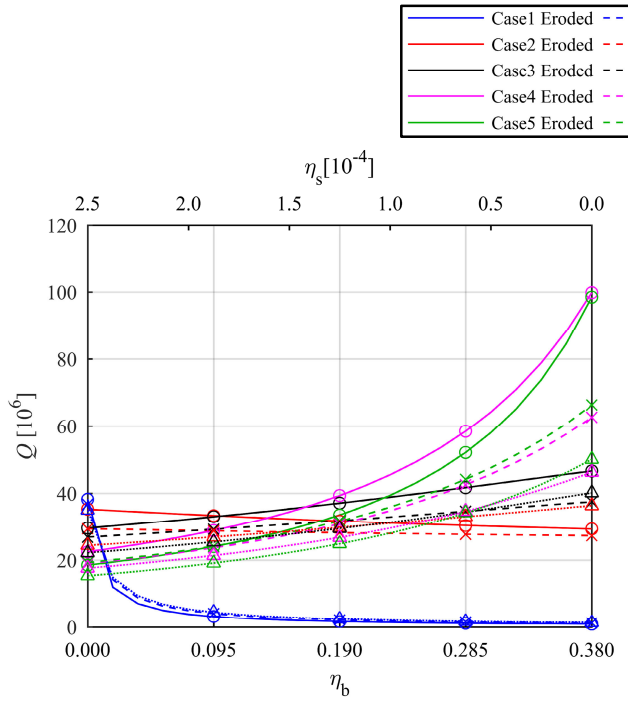

(a)

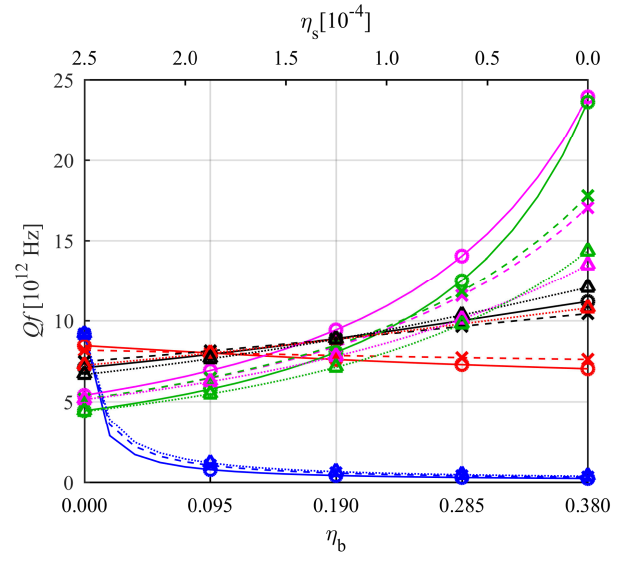

(c) $\eta_{s}\left[10^{-4}\right]$

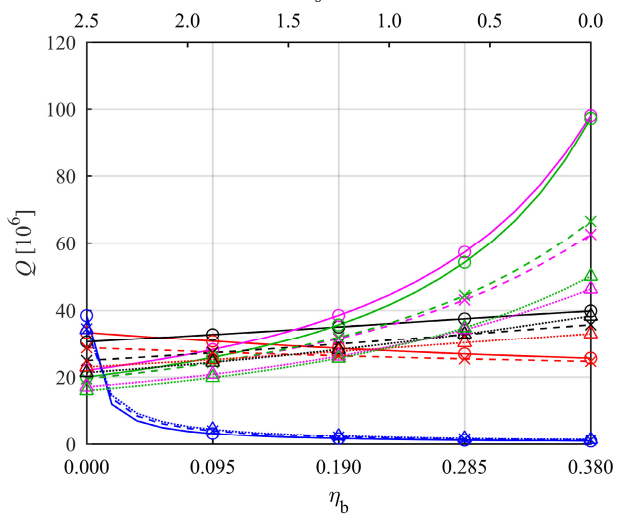

(b)

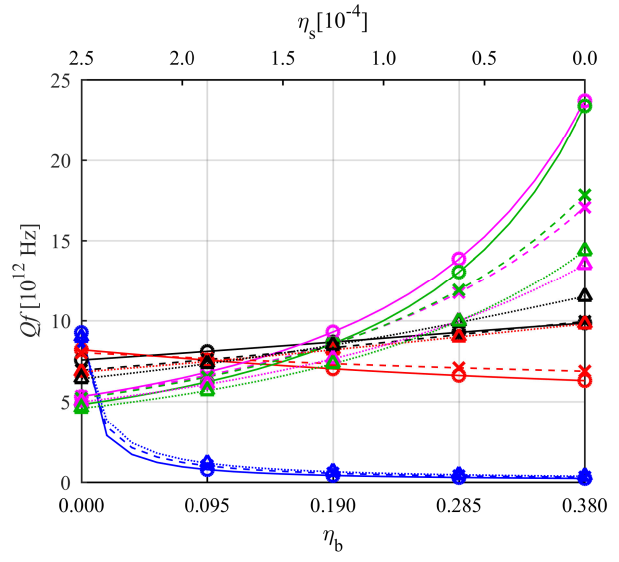

(d) 
Fig. 8. Performance of maximizing- $Q f$ designs under different damping combinations: (a) and (c) at $\beta_{\mathrm{f}}=$ 120 without post-processing; (b) and (d) re-evaluation at $\beta_{\mathrm{f}}=300$.

\section{Conclusion}

This paper employs a linearized simplified shell model, which benefits optimization and captures essential physics, to model and optimize high-stress membrane systems. Topology optimization is conducted to enhance the quality factor $Q$ and frequency quality factor $Q f$ in the fundamental mode. The conclusions are the following:

(1) $Q$ and $Q f$ of high-stress membranes are highly sensitive to structural geometry. The upper bound for the increment of design variables should be small enough, and fine mesh should be a basic requirement for finite element models.

(2) The robust formulation employed in this study partly introduces robustness to manufacturing variations into the designs, partly helps in getting rid of gray regions, and partly introduces a stricter length-scale. Hence, the obtained designs arising from the robust formulation are pretty robust and deliver the expected response independent of small manufacturing variations, provided that the underlying damping sources are sufficiently captured.

(3) The geometry of the optimized design closely relates to the damping mechanism. The optimizations simultaneously considering different damping sources generate results subjected to less influence from damping variations.

(4) All the optimized designs, which have not been devised by physicists and tested in laboratories so far, exhibit fundamental modes with frequency-quality factors sufficient to enter the optomechanical quantum regime at room temperature. This demonstrates that topology optimization is a useful and powerful tool to design functional structures beyond existing design experience.

\section{Acknowledgements}

The authors wish to thank all the members in TOPOPT group for fruitful discussions. The use of the MMA code from Professor Krister Svanberg (Department of Mathematics, KTH Royal Institute of Technology) and the constructive suggestions from Professor Xilin Lu (College of Civil Engineering, Tongji University) are much appreciated. The authors also appreciate the meaningful discussion with Dennis Høj (Department of Physics, Technical University of Denmark). Wenjun Gao acknowledges China Scholarship Council (CSC) for financially supporting his study at Technical University of Denmark from 2017 to 2019. Fengwen Wang and Ole Sigmund acknowledge the support from the Villum Foundation through the Villum Investigator Project InnoTop.

\section{Appendix}

\section{Derivation of sensitivity}

Necessary derivatives required by the gradient-based optimizer is concisely derived. A similar derivation is also found in [20]. Taking the derivative of $f, Q$ or $Q f$ with respect to a design variable $\rho_{e}$ yields the terms including the derivative of $\boldsymbol{U}_{0}$ with respect to $\rho_{e}$. The adjoint method, see Ref. [45] for a review, is employed to avoid solving $\mathrm{d} \boldsymbol{U}_{0} / \mathrm{d} \rho_{e}$ in order to reduce computational cost. Taking the derivative of (1) and (2) with respect to a projected physical density $\bar{\rho}_{e}$ yields

$$
\left(\boldsymbol{K}_{\mathrm{t}}+\mathrm{i} \boldsymbol{C}-\lambda_{j} \boldsymbol{M}\right) \frac{\mathrm{d} \boldsymbol{\phi}_{j}}{\mathrm{~d} \bar{\rho}_{e}}+\frac{\mathrm{d}\left(\boldsymbol{K}_{\mathrm{t}}+\mathrm{i} \boldsymbol{C}-\lambda_{j} \boldsymbol{M}\right)}{\mathrm{d} \bar{\rho}_{e}} \boldsymbol{\phi}_{j}=\mathbf{0}
$$




$$
\frac{\mathrm{d} \boldsymbol{F}_{\sigma}}{\mathrm{d} \bar{\rho}_{e}}-\frac{\mathrm{d} \boldsymbol{K}_{0}}{\mathrm{~d} \bar{\rho}_{e}} \boldsymbol{U}_{0}-\boldsymbol{K}_{0} \frac{\mathrm{d} \boldsymbol{U}_{0}}{\mathrm{~d} \bar{\rho}_{e}}=\mathbf{0}
$$

After eliminating the first term in (18) through left-multiplying by $\boldsymbol{\phi}_{j}^{\mathrm{T}}$, the second term of (18) is augmented by (19) to arrive at

$$
\boldsymbol{\phi}_{j}^{\mathrm{T}} \frac{\mathrm{d}\left(\boldsymbol{K}_{0}+\boldsymbol{K}_{\sigma}+\mathrm{i} \boldsymbol{C}-\lambda_{j} \boldsymbol{M}\right)}{\mathrm{d} \bar{\rho}_{e}} \boldsymbol{\phi}_{j}+\boldsymbol{\beta}^{\mathrm{T}}\left(\frac{\mathrm{d} \boldsymbol{F}_{\sigma}}{\mathrm{d} \bar{\rho}_{e}}-\frac{\mathrm{d} \boldsymbol{K}_{0}}{\mathrm{~d} \bar{\rho}_{e}} \boldsymbol{U}_{0}-\boldsymbol{K}_{0} \frac{\mathrm{d} \boldsymbol{U}_{0}}{\mathrm{~d} \bar{\rho}_{e}}\right)=\mathbf{0}
$$

where $\boldsymbol{\beta}$ is the adjoint vector and $\boldsymbol{K}_{\sigma}$ should be interpreted as a function of $\bar{\rho}_{e}$ and $\boldsymbol{U}_{0}$. Note that $\mathrm{d} \boldsymbol{K}_{\mathrm{B}} / \mathrm{d} \bar{\rho}_{e}=$ $\mathbf{0}$. The derivative of $\boldsymbol{K}_{\sigma}$ with respect to $\bar{\rho}_{e}$ contains two parts as

$$
\frac{\mathrm{d} \boldsymbol{K}_{\sigma}}{\mathrm{d} \bar{\rho}_{e}}=\frac{\partial \boldsymbol{K}_{\sigma}}{\partial \boldsymbol{U}_{0}} \frac{\mathrm{d} \boldsymbol{U}_{0}}{\mathrm{~d} \bar{\rho}_{e}}+\frac{\partial \boldsymbol{K}_{\sigma}}{\partial \bar{\rho}_{e}}
$$

Substituting from (21) into (20) yields

$$
\begin{aligned}
\frac{\mathrm{d} \lambda_{j}}{\mathrm{~d} \bar{\rho}_{e}} \boldsymbol{\phi}_{j}^{\mathrm{T}} \boldsymbol{M} \boldsymbol{\phi}_{j}=\boldsymbol{\phi}_{j}^{\mathrm{T}}\left(\frac{\mathrm{d} \boldsymbol{K}_{0}}{\mathrm{~d} \bar{\rho}_{e}}+\mathrm{i} \frac{\mathrm{d} \boldsymbol{C}}{\mathrm{d} \bar{\rho}_{e}}+\frac{\partial \boldsymbol{K}_{\sigma}}{\partial \bar{\rho}_{e}}-\lambda_{j} \frac{\mathrm{d} \boldsymbol{M}}{\mathrm{d} \bar{\rho}_{e}}\right) \boldsymbol{\phi}_{j}+\left(\boldsymbol{\phi}_{j}^{\mathrm{T}} \frac{\partial\left(\boldsymbol{K}_{\sigma} \boldsymbol{\phi}_{j}\right)}{\partial \boldsymbol{U}_{0}}-\boldsymbol{\beta}^{\mathrm{T}} \boldsymbol{K}_{0}\right) \frac{\mathrm{d} \boldsymbol{U}_{0}}{\mathrm{~d} \bar{\rho}_{e}} \\
+\boldsymbol{\beta}^{\mathrm{T}}\left(\frac{\mathrm{d} \boldsymbol{F}_{\sigma}}{\mathrm{d} \bar{\rho}_{e}}-\frac{\mathrm{d} \boldsymbol{K}_{0}}{\mathrm{~d} \bar{\rho}_{e}} \boldsymbol{U}_{0}\right)
\end{aligned}
$$

In order to eliminate the term containing the implicit derivative of displacements, namely $\mathrm{d} \boldsymbol{U}_{0} / \mathrm{d} \bar{\rho}_{e}$, the adjoint equation (16) should be solved. Since $\lambda_{j}=\left(\omega_{j}\right)^{2}=\left(\omega_{\mathrm{r}, j}+\mathrm{i} \omega_{\mathrm{s}, j}\right)^{2}$, the derivatives of $\omega_{\mathrm{r}, j}$ and $\omega_{\mathrm{s}, j}$ with respect to design variables can be expressed as

$$
\begin{gathered}
\frac{\mathrm{d} \omega_{j}}{\mathrm{~d} \rho_{e}}=\frac{1}{2 \sqrt{\lambda_{j}}} \frac{\mathrm{d} \lambda_{j}}{\mathrm{~d} \rho_{e}} \\
\frac{\mathrm{d} \omega_{\mathrm{r}, j}}{\mathrm{~d} \rho_{e}}=\operatorname{real}\left(\frac{\mathrm{d} \omega_{j}}{\mathrm{~d} \rho_{e}}\right) \\
\frac{\mathrm{d} \omega_{\mathrm{s}, j}}{\mathrm{~d} \rho_{e}}=\operatorname{imag}\left(\frac{\mathrm{d} \omega_{j}}{\mathrm{~d} \rho_{e}}\right) \\
\frac{\mathrm{d} Q_{j}}{\mathrm{~d} \rho_{e}}=\frac{1}{2}\left(\frac{\omega_{\mathrm{s}, j} \frac{\mathrm{d} \omega_{\mathrm{r}, j}}{\mathrm{~d} \rho_{e}}-\omega_{\mathrm{r}, j} \frac{\mathrm{d} \omega_{\mathrm{s}, j}}{\mathrm{~d} \rho_{e}}}{\omega_{\mathrm{s}, j}^{2}}\right)
\end{gathered}
$$




\section{References}

[1] A.G. Krause, et al., A high-resolution microchip optomechanical accelerometer, Nature Photonics 6 (2012) 768-772.

[2] M. Yuan, V. Singh, Y.M. Blanter, G.A. Steele, Large cooperativity and microkelvin cooling with a three-dimensional optomechanical cavity, Nature Communications 6 (2015) 8491.

[3] R. Andrews, R. Peterson, et al., Bidirectional and efficient conversion between microwave and optical light, Nature Physics 10 (2014) 321-326.

[4] J. Thompson, B. Zwick, et al., Strong dispersive coupling of a high-finesse cavity to a micromechanical membrane, Nature 452 (2008) 72-75.

[5] J.D. Teufel, et al., Sideband cooling of micromechanical motion to the quantum ground state, Nature 475 (2011) 359-363.

[6] J. Chan, et al., Laser cooling of a nanomechanical oscillator into its quantum ground state, Nature 478 (2011) 89-92.

[7] F. Marquardt, J.P. Chen, A.A. Clerk, S.M. Girvin, Quantum theory of cavity-assisted sideband cooling of mechanical motion, Physical Review Letters 99 (2007) 093902.

[8] M. Aspelmeyer, T.J. Kippenberg, F. Marquardt, Cavity optomechanics, Reviews of Modern Physics 86(4) (2014) 1391.

[9] S. Schmid, K.D. Jensen, K.H. Nielsen, A. Boisen, Damping mechanisms in high-Q micro and nanomechanical string resonators, Physical Review B 84 (2011) 165307.

[10] H. Kim, D.H. Shin, et al., Accurate and precise determination of mechanical properties of silicon nitride beam nanoelectromechanical devices, ACS Applied Materials \& Interfaces 9 (8) (2017) 7282-7287.

[11] S.S. Verbridge, D.F. Shapiro, H.G. Craighead, et al., Macroscopic tuning of nanomechanics: substrate bending for reversible control of frequency and quality factor of nanostring resonators, Nano Letters 7 (6) (2007) 1728-1735.

[12] Q.P. Unterreithmeier, et al., Damping of nanomechanical resonators, Physical Review Letters 105 (2010) 027205.

[13] P.L. Yu, T.P. Purdy, C.A. Regal, Control of material damping in high-Q membrane microresonators, Physical Review Letters 108 (2012) 083603.

[14] D.J. Wilson, C.A. Regal, S.B. Papp, H.J. Kimble, Cavity optomechanics with stoichiometric SiN films, Physical Review Letters 103 (2009) 207204.

[15] S. Chakram, Y.S. Patil, L. Chang, M. Vengalattore, Dissipation in ultrahigh quality factor SiN membrane resonators, Physical Review Letter 112 (2014) 127201.

[16] C. Reinhardt, T. Muller, A. Bourassa, J.C. Sankey, Ultralow-noise SiN trampoline resonators for sensing and optomechanics, Physical Review X 6 (2016) 021001.

[17] R. Norte, J.P. Moura, S. Gröblacher, Mechanical resonators for quantum optomechanics experiments at room temperature, Physical Review Letter 116 (2016) 147202.

[18] R.N. Candler, et al., Impact of geometry on thermoelastic dissipation in micromechanical resonant beams, Journal of Microelectromechanical Systems 5 (4) (2006) 927-933.

[19] M.P. Bendsøe, N. Kikuchi, Generating optimal topologies in structural design using a homogenization method, Computer Methods in Applied Mechanics and Engineering 71 (2) (1988) 197-224.

[20] N.L. Pedersen, On topology optimization of plates with prestress, International Journal for Numerical Methods in Engineering 51 (2) (2001) 225-239.

[21] A.A. Larsen, B. Laksafoss, J.S. Jensen, O. Sigmund, Topological material layout in plates for vibration suppression and wave propagation control, Structural and Multidisciplinary Optimization 37 (6) (2009) 585-594.

[22] D.D. Gerrard, Y. Chen, S.A. Chandorkar, G. Yu, et al., Topology optimization for reduction of thermoelastic dissipation in MEMS resonators, in: 2017 19th International Conference on Solid-State Sensors, Actuators and Microsystems (TRANSDUCERS), IEEE, Kaohsiung, Taiwan, 2017, pp. 794-797. 
[23] X. Liang, S.G. Johnson, Formulation for scalable optimization of microcavities via the frequencyaveraged local density of states, Optics Express 21 (25) (2013) 30812-30841.

[24] F. Wang, R.E. Christiansen, Y. Yu, J. Mørk, O. Sigmund, Maximizing the quality factor to mode volume ratio for ultra-small photonic crystal cavities, Applied Physics Letters 113 (2018) 241101.

[25] Y. Fu, L. Li, Y. Hu, Enlarging quality factor in microbeam resonators by topology optimization, Journal of Thermal Stresses 42 (3) (2019) 341-360.

[26] D. Kleckner, et al., Optomechanical trampoline resonators, Optics Express 19 (24) (2011) 1970819716.

[27] E.N. Dvorkin, K.J. Bathe, A continuum mechanics based four-node shell element for general non-linear analysis, Engineering Computations 1 (1) (1984) 77-88.

[28] K.J. Bathe, Finite Element Procedures, Prentice hall, New Jersey, 1996.

[29] S. Schmid, L.G. Villanueva, M.L. Roukes, Fundamentals of Nanomechanical Resonators, Springer, Switzerland, 2016.

[30] Y. Tsaturyan, A. Barg, E.S. Polzik, A. Schliesser, Ultracoherent nanomechanical resonators via soft clamping and dissipation dilution, Nature Nanotechnology 12 (2017) 776-783.

[31] M.P. Bendsøe, Optimal shape design as a material distribution problem, Structural Optimization 1 (4) (1989) 193-202.

[32] M. Zhou, G. Rozvany, The COC algorithm, Part II: topological, geometrical and generalized shape optimization, Computer Methods in Applied Mechanics and Engineering 89 (1-3) (1991) 309-336.

[33] O. Sigmund, A 99 line topology optimization code written in Matlab, Structural and Multidisciplinary Optimization 21 (2) (2001) 120-127.

[34] E. Andreassen, A. Clausen, M. Schevenels, B.S. Lazarov, O. Sigmund, Efficient topology optimization in matlab using 88 lines of code, Structural and Multidisciplinary Optimization 43 (1) (2011) 1-16.

[35] M. Stolpe, K. Svanberg, An alternative interpolation scheme for minimum compliance topology optimization, Structural and Multidisciplinary Optimization 22 (2) (2001) 116-124.

[36] F. Wang, B.S. Lazarov, O. Sigmund, On projection methods, convergence and robust formulations in topology optimization, Structural and Multidisciplinary Optimization 43 (6) (2011) 767-784.

[37] A.R. Diaz, N. Kikuchi, Solutions to shape and topology eigenvalue optimization problems using a homogenization method, International Journal for Numerical Methods in Engineering 35 (7) (1992) $1487-1502$.

[38] Z.D. Ma, N. Kikuchi, H.C. Cheng, Topological design for vibrating structures, Computer Methods in Applied Mechanics and Engineering 121 (1-4) (1995) 259-280.

[39] N.L. Pedersen, Maximization of eigenvalues using topology optimization, Structural and Multidisciplinary Optimization 20 (1) (2000) 2-11.

[40] M.P. Bendsøe, O. Sigmund, Topology Optimization - Theory, Methods and Applications, Springer, Berlin, 2003.

[41] J. Du, N. Olhoff, Topological design of freely vibrating continuum structures for maximum values of simple and multiple eigenfrequencies and eigenfrequency gaps, Structural and Multidisciplinary Optimization 34 (2) (2007) 91-110.

[42] F. Wang, B.S. Lazarov, O. Sigmund, J.S. Jensen, Interpolation scheme for fictitious domain techniques and topology optimization of finite strain elastic problems, Computer Methods in Applied Mechanics and Engineering 276 (2014) 453-472.

[43] O. Sigmund, Morphology-based black and white filters for topology optimization, Structural and Multidisciplinary Optimization 33 (4-5) (2007) 401-424.

[44] K. Svanberg, Method of moving asymptotes - a new method for structural optimization, International Journal for Numerical Methods in Engineering 24 (2) (1987) 359-373.

[45] D.A. Tortorelli, P. Michaleris, Design sensitivity analysis: overview and review, Inverse Problems in Engineering 1 (1) (1994) 71-105. 